\title{
Probing the application of kinetic theory to Mg-phyllosilicate growth with Si isotope doping
}

\section{ZHENGQIANG CHE $^{1}$, MICHAEL NIGHTINGALE ${ }^{1}$ AND BENJAMIN TUTOLO ${ }^{2}$}

${ }^{1}$ University of Calgary

${ }^{2}$ University of Calgary, Department of Geoscience

Presenting Author: zhengqiang.che@ucalgary.ca

The principle of detailed balance (PDB) played a defining role in the derivation of the widely-used Transition State Theory rate law equation and serves as an important link between geochemical kinetics and thermodynamics. Although significant strides have been made in applying the PDB to simple systems (e.g., $\mathrm{SiO}_{2}$-water), experimental verification of the $\mathrm{PDB}$ is lacking for more complex minerals such as the phyllosilicates. Here, we use ${ }^{29} \mathrm{Si}$ isotopic doping techniques to quantify the rates of reaction between amorphous $\mathrm{Mg}$-silicate and supersaturated solutions. The results show that the ratio of the forward and backward reaction rates approach unity as the saturation state of the solution approaches the apparent solubility of amorphous $\mathrm{Mg}$-silicate. Both precipitation rates coupled with equivalent dissolution rates and precipitation rates coupled with negligible dissolution rates appear to obey the same rate function over the degrees of supersaturation we explore, indicating that the elementary step limiting the rate of precipitation remains the same. Accordingly, our results demonstrates that the PDB is applicable to amorphous $\mathrm{Mg}$-silicate-water reactions, thereby reenforcing the use of TST rate equations to describe growth of Mg-phyllosilicates, and perhaps other phyllosilicates. The experimental data can also be taken as evidence that the apparent solubility of amorphous Mg-silicate, a concept previously explained using the kinetic theory of nucleation and growth, also has a thermodynamic meaning, in that it represents a quasiequilibrium with the poorly crystalline phase. The measured, non-negligible forward and backward rates suggest that, even in this quasi-equilibrium state where little if any net reaction is occurring, isotopic signatures can be reset. Moreover, the significant discrepancy between the heterogeneous growth rates (net reaction rates) on the amorphous $\mathrm{Mg}$-silicate substrate versus those measured on crystalline talc substrates indicates that mineral crystallinity likely plays a key role in mineral growth during diagenesis. 